\title{
Sharp Power Mean Bounds for the One-Parameter Harmonic Mean
}

\author{
Yu-Ming Chu, ${ }^{1}$ Li-Min Wu, ${ }^{2}$ and Ying-Qing Song ${ }^{1}$ \\ ${ }^{1}$ School of Mathematics and Computation Sciences, Hunan City University, Yiyang 413000, China \\ ${ }^{2}$ Department of Mathematics, Huzhou University, Huzhou 313000, China
}

Correspondence should be addressed to Yu-Ming Chu; chuyuming2005@126.com

Received 3 November 2014; Accepted 27 April 2015

Academic Editor: David R. Larson

Copyright (C) 2015 Yu-Ming Chu et al. This is an open access article distributed under the Creative Commons Attribution License, which permits unrestricted use, distribution, and reproduction in any medium, provided the original work is properly cited.

We present the best possible parameters $\alpha=\alpha(r)$ and $\beta=\beta(r)$ such that the double inequality $M_{\alpha}(a, b)<H_{r}(a, b)<M_{\beta}(a, b)$ holds for all $r \in(0,1 / 2)$ and $a, b>0$ with $a \neq b$, where $M_{p}(a, b)=\left[\left(a^{p}+b^{p}\right) / 2\right]^{1 / p}(p \neq 0)$ and $M_{0}(a, b)=\sqrt{a b}$ and $H_{r}(a, b)=$ $2[r a+(1-r) b][r b+(1-r) a] /(a+b)$ are the power and one-parameter harmonic means of $a$ and $b$, respectively.

\section{Introduction}

For $p \in \mathbb{R}$ and $a, b>0$, the $p$ th power mean $M_{p}(a, b)$ of $a$ and $b$ is defined by

$$
M_{p}(a, b)=\left(\frac{a^{p}+b^{p}}{2}\right)^{1 / p}
$$

$$
(p \neq 0), M_{0}(a, b)=\sqrt{a b} .
$$

It is well known that $M_{p}(a, b)$ is strictly increasing with respect to $p \in \mathbb{R}$ for fixed $a, b>0$ with $a \neq b$, symmetric and homogeneous of degree 1. Many classical means are special cases of the power mean: for example, $M_{-1}(a, b)=2 a b /(a+$ $b)=H(a, b)$ is the harmonic mean, $M_{0}(a, b)=\sqrt{a b}=G(a, b)$ is the geometric mean, $M_{1}(a, b)=(a+b) / 2=A(a, b)$ is the arithmetic mean, and $M_{2}(a, b)=\sqrt{\left(a^{2}+b^{2}\right) / 2}=Q(a, b)$ is the quadratic mean. The main properties of the power mean are given in [1]. Recently, the power mean has attracted the attention of many researchers. In particular, many remarkable inequalities for the power mean can be found in the literature [2-10].

Let $L(a, b)=(b-a) /(\log b-\log a), P(a, b)=(a-$ $b) /[2 \arcsin ((a-b) /(a+b))], I(a, b)=1 / e\left(b^{b} / a^{a}\right)^{1 /(b-a)}$, $T(a, b)=(a-b) /[2 \arctan ((a-b) /(a+b))]$, and $C(a, b)=$ $\left(a^{2}+b^{2}\right) /(a+b)$ be the logarithmic, first Seiffert, identric, second Seiffert, and contraharmonic means of two distinct positive real numbers $a$ and $b$, respectively. Then it is well known that the inequalities

$$
\begin{aligned}
H(a, b) & <G(a, b)<L(a, b)<P(a, b)<I(a, b) \\
& <A(a, b)<T(a, b)<Q(a, b)<C(a, b)
\end{aligned}
$$

hold for all $a, b>0$ with $a \neq b$.

Lin [11] proved that the double inequality

$$
M_{p}(a, b)<L(a, b)<M_{q}(a, b)
$$

holds for all $a, b>0$ with $a \neq b$ if and only if $p \leq 0$ and $q \geq 1 / 3$.

In [12], Pittenger presented the best possible parameters $\lambda=\lambda(p)$ and $\mu=\mu(p)$ such that the double inequality

$$
M_{\lambda}(a, b)<L_{p}(a, b)<M_{\mu}(a, b)
$$

holds for all $a, b>0$ with $a \neq b$, where $L_{p}(a, b)=\left[\left(b^{p+1}-\right.\right.$ $\left.\left.a^{p+1}\right) /((p+1)(b-a))\right]^{1 / p}(p \neq 0,-1), L_{0}(a, b)=I(a, b)$, and $L_{-1}(a, b)=L(a, b)$ is the generalized logarithmic mean of $a$ and $b$.

Jagers [13] and Seiffert [14] proved that the double inequalities

$$
\begin{gathered}
M_{1 / 2}(a, b)<P(a, b)<M_{2 / 3}(a, b), \\
M_{1}(a, b)<T(a, b)<M_{2}(a, b)
\end{gathered}
$$

hold for all $a, b>0$ with $a \neq b$. 
In $[15,16]$, the authors proved that the double inequalities

$$
\begin{aligned}
M_{\log 2 /(1+\log 2)}(a, b) & <\frac{I(a, b)+L(a, b)}{2}<M_{1 / 2}(a, b), \\
M_{0}(a, b) & <\sqrt{I(a, b) L(a, b)}<M_{1 / 2}(a, b)
\end{aligned}
$$

hold for all $a, b>0$ with $a \neq b$.

Costin and Toader [17] proved that the double inequality

$$
M_{4 / 3}(a, b)<T(a, b)<M_{5 / 3}(a, b)
$$

holds for all $a, b>0$ with $a \neq b$.

In [18-20], the authors proved that the double inequalities

$$
\begin{aligned}
& M_{p}(a, b)<P(a, b)<M_{q}(a, b), \\
& M_{r}(a, b)<T(a, b)<M_{s}(a, b)
\end{aligned}
$$

hold for all $a, b>0$ with $a \neq b$ if and only if $p \leq \log \pi / \log 2$, $q \geq 2 / 3, r \leq \log 2 /(\log \pi-\log 2)$, and $s \geq 5 / 3$.

Čizmesija [21] proved that $p=-\alpha / 2$ and $q=$ $\log 2 /[\log 4-\log (1-\alpha)]$ are the best possible parameters such that the double inequality $M_{p}(a, b)<[H(a, b)+\alpha G(a, b)+(1-$ $\alpha) A(a, b)] / 2<M_{q}(a, b)$ holds for all $\alpha \in(0,1)$ and $a, b>0$ with $a \neq b$.

In $[22,23]$, the authors proved that the inequalities

$$
\begin{aligned}
& M_{p}[\mathscr{K}(r), \mathscr{E}(r)]>\frac{\pi}{2}, \\
& M_{\lambda}\left[\mathscr{K}(r), \mathscr{K}\left(\sqrt{1-r^{2}}\right)\right] \\
& \quad \leq \mathscr{K}\left(\frac{\sqrt{2}}{2}\right) \leq M_{\mu}\left[\mathscr{K}(r), \mathscr{K}\left(\sqrt{1-r^{2}}\right)\right]
\end{aligned}
$$

hold for all $r \in(0,1)$ if and only if $p \geq-1 / 2, \lambda \leq$ $\log 2 /(\log \pi-\log 2)-\log [\mathscr{K}(\sqrt{2} / 2)]=-4.18, \ldots$, and $\mu \geq 1-4 \mathscr{K}^{4}(\sqrt{2} / 2) / \pi^{2}=-3.789, \ldots$, where $\mathscr{K}(r)=$ $\int_{0}^{\pi / 2}\left(1-r^{2} \sin ^{2} \theta\right)^{-1 / 2} d \theta$ and $\mathscr{E}(r)=\int_{0}^{\pi / 2}\left(1-r^{2} \sin ^{2} \theta\right)^{1 / 2} d \theta$ are, respectively, the complete elliptic integrals of the first and second kinds.

Let $p \in[0,1]$ and $N$ be the bivariate symmetric mean. Then, the one-parameter mean $N_{p}(a, b)$ was defined by Neuman [24] as follows:

$$
N_{p}(a, b)=N[p a+(1-p) b, p b+(1-p) a] .
$$

Let $p_{1}, q_{1}, p_{2}, q_{2}, \lambda_{1}, \lambda_{2}, \lambda_{3}, \lambda, \mu \in(0,1 / 2)$ and $p_{3}, q_{3} \in$ $(1 / 2,1)$. Then, the authors in [25-28] proved that the inequalities

$$
\begin{aligned}
& H_{p_{1}}(a, b)<P(a, b)<H_{q_{1}}(a, b), \\
& L_{p_{2}}(a, b)<P(a, b)<L_{q_{2}}(a, b), \\
& H_{\lambda_{1}}(a, b)>G(a, b), \\
& H_{\lambda_{2}}(a, b)>L(a, b), \\
& G_{\lambda_{3}}(a, b)>L(a, b), \\
& C_{p_{3}}(a, b)<T(a, b)<C_{q_{3}}(a, b), \\
& G_{\lambda}(a, b)<I(a, b)<G_{\mu}(a, b)
\end{aligned}
$$

hold for all $a, b>0$ with $a \neq b$ if and only if $p_{1} \leq 1 / 2-$ $\sqrt{1-2 / \pi} / 2, q_{1} \geq 1 / 2-\sqrt{6} / 12, p_{2} \leq \lambda_{0}, q_{2} \geq 1 / 2-\sqrt{2} / 4$, $\lambda_{1} \geq 1 / 2-\sqrt{2} / 4, \lambda_{2} \geq 1 / 2-\sqrt{3} / 6, \lambda_{3} \geq 1 / 2-\sqrt{6} / 6, p_{3} \leq$ $1 / 2+\sqrt{4 / \pi-1} / 2, q_{3} \geq 1 / 2+\sqrt{3} / 6, \lambda \leq 1 / 2-\sqrt{1-4 / e^{2}} / 2$, and $\mu \geq 1 / 2-\sqrt{3} / 6$, where $\lambda_{0}$ is the unique solution of the equation $\log [(1-\lambda) / \lambda]=\pi(1-2 \lambda)$.

The main purpose of this paper is to present the best possible parameters $\alpha=\alpha(r)$ and $\beta=\beta(r)$ such that the double inequality $M_{\alpha}(a, b)<H_{r}(a, b)<M_{\beta}(a, b)$ holds for all $r \in(0,1 / 2)$ and $a, b>0$ with $a \neq b$.

\section{Lemmas}

In order to prove our main result we need three lemmas, which we present in this section.

Lemma 1. The inequality

$$
-8 r^{2}+8 r-1+\frac{\log 2}{\log [2 r(1-r)]}<0
$$

holds for all $r \in(0,1 / 2)$.

Proof. It is not difficult to verify that $\log [2 r(1-r)]<0$ for all $r \in(0,1 / 2)$. Therefore, we only need to prove that $f(r)>0$ for $r \in(0,1 / 2)$, where $f(r)=\left(-8 r^{2}+8 r-1\right) \log [2 r(1-r)]+\log 2$. Simple computations lead to

$$
\begin{aligned}
f\left(\frac{1}{2}\right) & =0, \\
f^{\prime}(r) & =(1-2 r) g(r),
\end{aligned}
$$

where

$$
\begin{aligned}
g(r) & =8 \log [2 r(1-r)]-\frac{1}{r(1-r)}+8, \\
g\left(\frac{1}{2}\right) & =4(1-\log 4)<0 \\
g^{\prime}(r) & =\frac{(1-2 r)[8 r(1-r)+1]}{r^{2}(1-r)^{2}}>0
\end{aligned}
$$

for all $r \in(0,1 / 2)$.

Inequality (15) implies that $g(r)<0$ for all $r \in(0,1 / 2)$. Then, from (13) we clearly see that $f(r)>0$ for all $r \in(0,1 / 2)$.

Lemma 2. The inequality

$$
\begin{aligned}
& -16 r^{2}+16 r-2-\left(8 r^{2}-8 r-1\right) \frac{\log 2}{\log [2 r(1-r)]} \\
& +\left[\frac{\log 2}{\log [2 r(1-r)]}\right]^{2}<0
\end{aligned}
$$

holds for all $r \in(0,1 / 2)$.

Proof. Let $p=-\log 2 / \log [2 r(1-r)]$ and $q=-8 r^{2}+8 r-1$. Then, it is not difficult to verify that

$$
0<p<1
$$

for all $r \in(0,1 / 2)$. 
It follows from Lemma 1 that

$$
p>q
$$

for all $r \in(0,1 / 2)$.

Inequalities (17) and (18) lead to

$$
\begin{aligned}
- & 16 r^{2}+16 r-2-\left(8 r^{2}-8 r-1\right) \frac{\log 2}{\log [2 r(1-r)]} \\
+ & {\left[\frac{\log 2}{\log [2 r(1-r)]}\right]^{2}=2 q-(q+2) p+p^{2} } \\
& =-(p-q)(2-p)<0
\end{aligned}
$$

for all $r \in(0,1 / 2)$.

Lemma 3. The inequality

$$
\begin{aligned}
& -6 r^{2}+6 r-\left(12 r^{2}-12 r+1\right) \frac{\log 2}{\log [2 r(1-r)]} \\
& +\left[\frac{\log 2}{\log [2 r(1-r)]}\right]^{2}>0
\end{aligned}
$$

holds for all $r \in(0,1 / 2)$.

Proof. Let $p=-\log 2 / \log [2 r(1-r)]$ and $q=-8 r^{2}+8 r-1$. Then, it follows from (17) and (18) that

$$
\begin{aligned}
& -6 r^{2}+6 r-\left(12 r^{2}-12 r+1\right) \frac{\log 2}{\log [2 r(1-r)]} \\
& +\left[\frac{\log 2}{\log [2 r(1-r)]}\right]^{2}=\frac{3}{4}(q+1) \\
& +\left[1-\frac{3}{2}(q+1)\right] p+p^{2}=\frac{3}{4}(q+1)(1-2 p) \\
& +p(p+1)>\frac{3}{4}(q+1)(1-2 p)+p(q+1) \\
& \quad=\frac{1}{4}(q+1)(3-2 p)=2 r(1-r)(3-2 p)>0
\end{aligned}
$$

for all $r \in(0,1 / 2)$.

\section{Main Results}

Theorem 4. The double inequality

$$
M_{\alpha}(a, b)<H_{r}(a, b)<M_{\beta}(a, b)
$$

holds for all $r \in(0,1 / 2)$ and $a, b>0$ with $a \neq b$ if and only if $\alpha \leq-8 r^{2}+8 r-1$ and $\beta \geq-\log 2 / \log [2 r(1-r)]$.

Proof. Without loss of generality, we assume that $a=x \in$ $(1, \infty)$ and $b=1$. Let $\log \left[H_{r}(x, 1)\right]-\log \left[M_{p}(x, 1)\right]=f(x)$, where

$$
\begin{aligned}
f(x)= & \log \frac{r(1-r) x^{2}+\left(1-2 r+2 r^{2}\right) x+r(1-r)}{1+x} \\
& +\frac{(1+p) \log 2-\log \left(1+x^{p}\right)}{p} .
\end{aligned}
$$

Then, simple computations lead to

$$
f(1)=0 \text {, }
$$

$$
f^{\prime}(x)
$$

$$
=\frac{g(x)}{\left[r(1-r) x^{2}+\left(1-2 r+2 r^{2}\right) x+r(1-r)\right](1+x)\left(1+x^{p}\right) x^{1-p}},
$$

where

$$
\begin{aligned}
g(x)= & \left(-r^{2}+r\right) x^{3-p}+\left(-2 r^{2}+2 r\right) x^{2-p} \\
& +\left(3 r^{2}-3 r+1\right) x^{1-p} \\
& +\left(-3 r^{2}+3 r-1\right) x^{2}+\left(2 r^{2}-2 r\right) x \\
& +r^{2}-r, \\
g(1)= & 0, \\
g^{\prime}(1)= & -8 r^{2}+8 r-1-p, \\
g^{\prime \prime}(1)= & -16 r^{2}+16 r-2+p\left(8 r^{2}-8 r-1\right)+p^{2}, \\
g^{\prime \prime \prime}(x)= & (1-p) x^{-p-2} h(x),
\end{aligned}
$$

where

$$
\begin{aligned}
h(x)= & \left(-r^{2}+r\right)(3-p)(2-p) x^{2} \\
& -\left(-2 r^{2}+2 r\right) p(2-p) x \\
& +\left(3 r^{2}-3 r+1\right) p(p+1), \\
h(1)= & 6\left(-r^{2}+r\right)+p\left(12 r^{2}-12 r+1\right)+p^{2}, \\
h^{\prime}(x)= & 2\left(r-r^{2}\right)(2-p)[(3-p) x-p] .
\end{aligned}
$$

We divide the proof into two cases.

Case $1\left(p=-8 r^{2}+8 r-1\right)$. We divide the discussion into two subcases.

Subcase $1.1\left(p=-8 r^{2}+8 r-1\right.$ and $r \in(0,(2-\sqrt{2}) / 4) \cup(2-$ $\sqrt{2}) / 4,1 / 2))$. Then, we clearly see that $p \in(-1,0) \cup(0,1)$, and (28), (29), (32), and (33) lead to

$$
\begin{aligned}
g^{\prime}(1) & =g^{\prime \prime}(1)=0 \\
h(1) & =\frac{1}{4}(p+1)(3-2 p)>0 \\
h^{\prime}(x) & =\frac{1}{4}(p+1)(2-p)[(3-p) x-p] \\
& >\frac{1}{4}(p+1)(2-p)(3-2 p)>0
\end{aligned}
$$

for $x \in(1, \infty)$.

It follows easily from (24), (25), (27), (30), and (34)-(36) that

$$
H_{r}(x, 1)>M_{-8 r^{2}+8 r-1}(x, 1)
$$

for all $r \in(0,1 / 2)$. 
Subcase $1.2\left(p=-8 r^{2}+8 r-1\right.$ and $\left.r=(2-\sqrt{2}) / 4\right)$. Then, $p=0$, and (37) follows from

$$
\begin{aligned}
& H_{r}(x, 1)-M_{-8 r^{2}+8 r-1}(x, 1) \\
& \quad=\frac{x^{2}+6 x+1}{4(1+x)}-\sqrt{x}=\frac{(\sqrt{x}-1)^{2}}{4(1+x)} .
\end{aligned}
$$

Case $2(p=-\log 2 / \log [2 r(1-r)])$. Then, we clearly see that $p \in(0,1)$, and Lemmas 1-3 and (23), (26), (28), (29), and (32) lead to

$$
\begin{aligned}
\lim _{x \rightarrow+\infty} f(x) & =0, \\
\lim _{x \rightarrow+\infty} g(x) & =+\infty, \\
\lim _{x \rightarrow+\infty} g^{\prime}(x) & =+\infty, \\
g^{\prime}(1) & <0, \\
\lim _{x \rightarrow+\infty} g^{\prime \prime}(x) & =+\infty, \\
g^{\prime \prime}(1) & <0, \\
h(1) & >0
\end{aligned}
$$

and (36) again holds.

It follows from (30), (36), and (45) that $g^{\prime \prime}$ is strictly increasing on $(1, \infty)$. Then, (43) and (44) lead to the conclusion that there exists $\mu_{0}>1$ such that $g^{\prime}$ is strictly decreasing on $\left(1, \mu_{0}\right]$ and strictly increasing on $\left[\mu_{0}, \infty\right)$.

From (41) and (42) together with the piecewise monotonicity of $g^{\prime}$ we clearly see that there exists $\mu_{1}>1$ such that $g$ is strictly decreasing on $\left(1, \mu_{1}\right]$ and strictly increasing on $\left[\mu_{1}, \infty\right)$. Then, (25), (27), and (40) lead to the conclusion that there exists $\mu_{2}>1$ such that $f$ is strictly decreasing on $\left(1, \mu_{2}\right]$ and strictly increasing on $\left[\mu_{2}, \infty\right)$. Therefore,

$$
H_{r}(x, 1)<M_{-\log 2 / \log [2 r(1-r)]}(x, 1)
$$

for all $r \in(0,1 / 2)$ follows from (24) and (39) together with the piecewise monotonicity of $f$.

Next, we prove that $\alpha=-8 r^{2}+8 r-1$ and $\beta=$ $-\log 2 / \log [2 r(1-r)]$ are the best possible parameters such that the double inequality

$$
M_{\alpha}(a, b)<H_{r}(a, b)<M_{\beta}(a, b)
$$

holds for all $r \in(0,1 / 2)$ and $a, b>0$ with $a \neq b$.

Let $r \in(0,1 / 2), \alpha_{0}=-8 r^{2}+8 r-1, \beta_{0}=-\log 2 / \log [2 r(1-$ $r)], \varepsilon \in\left(0, \beta_{0}\right)$, and $t>0$. Then, we have

$$
\begin{aligned}
& H_{r}(1,1+t)-M_{\alpha_{0}+\varepsilon}(1,1+t) \\
& =\frac{2[(1-r)(1+t)+r][r(1+t)+1-r]}{t+2} \\
& -\left[\frac{1+(1+t)^{\alpha_{0}+\varepsilon}}{2}\right]^{1 /\left(\alpha_{0}+\varepsilon\right)}, \\
& \lim _{t \rightarrow+\infty} \frac{H_{r}(1, t)}{M_{\beta_{0}-\varepsilon}(1, t)}=2^{\varepsilon /\left[\beta_{0}\left(\beta_{0}-\varepsilon\right)\right]}>1 .
\end{aligned}
$$

Let $t \rightarrow 0^{+}$and make use of the Taylor expansion; then, (48) leads to

$$
H_{r}(1,1+t)-M_{\alpha_{0}+\varepsilon}(1,1+t)=-\frac{\varepsilon}{8} x^{2}+o\left(x^{2}\right) .
$$

Inequality (49) and equation (50) imply that for any $r \in$ $(0,1 / 2)$ and $\varepsilon \in\left(0, \beta_{0}\right)$ there exist $T=T(\varepsilon)>1$ and $\delta=$ $\delta(\varepsilon) \in(0,1)$ such that $H_{r}(1, t)>M_{\beta_{0}-\varepsilon}(1, t)$ for $t>T$ and $H_{r}(1,1+t)<M_{\alpha_{0}+\varepsilon}(1,1+t)$ for $t \in(0, \delta)$.

\section{Conflict of Interests}

The authors declare that there is no conflict of interests regarding the publication of this paper.

\section{Acknowledgments}

The research was supported by the Natural Science Foundation of China under Grants 61374086 and 11371125, the Natural Science Foundation of Zhejiang Province under Grant LY13A010004, and the Natural Science Foundation of Hunan Province under Grant 14JJ2127.

\section{References}

[1] P. S. Bullen, D. S. Mitrinović, and P. M. Vasić, Means and Their Inequalities, D. Reidel Publishing Company, Dordrecht, The Netherlands, 1988.

[2] C. O. Imoru, "The power mean and the logarithmic mean," International Journal of Mathematics and Mathematical Sciences, vol. 5, no. 2, pp. 337-343, 1982.

[3] J. E. Pečarić, "Generalization of the power means and their inequalities," Journal of Mathematical Analysis and Applications, vol. 161, no. 2, pp. 395-404, 1991.

[4] D. Lukkassen, "Means of power type and their inequalities," Mathematische Nachrichten, vol. 205, pp. 131-147, 1999.

[5] Z. Liu, "Remark on inequalities between Hölder and Lehmer means," Journal of Mathematical Analysis and Applications, vol. 247, no. 1, pp. 309-313, 2000.

[6] H. Alzer, "A power mean inequality for the gamma function," Monatshefte für Mathematik, vol. 131, no. 3, pp. 179-188, 2000.

[7] C. Mortici, "Arithmetic mean of values and value at mean of arguments for convex functions," The ANZIAM Journal, vol. 50, no. 1, pp. 137-141, 2008.

[8] C. Mortici, "A power series approach to some inequalities," The American Mathematical Monthly, vol. 119, no. 2, pp. 147-151, 2012.

[9] L.-M. Zhou, S.-L. Qiu, and F. Wang, "Inequalities for the generalized elliptic integrals with respect to Hölder means," Journal of Mathematical Analysis and Applications, vol. 386, no. 2, pp. 641-646, 2012.

[10] B. A. Bhayo, "On the power mean inequality of the hyperbolic metric of unit ball," The Journal of Prime Research in Mathematics, vol. 8, pp. 45-50, 2012.

[11] T. P. Lin, "The power mean and the logarithmic mean," The American Mathematical Monthly, vol. 81, pp. 879-883, 1974.

[12] A. O. Pittenger, "Inequalities between arithmetic and logarithmic means," Univerzitet u Beogradu. Publikacije Elektrotehničkog Fakulteta. Serija Matematika i Fizika, vol. 678-715, pp. 15-18, 1980. 
[13] A. A. Jagers, "Solutions of problem 887," Nieuw Archief voor Wiskunde, vol. 12, no. 4, pp. 230-231, 1994.

[14] H. J. Seiffert, “Aufgabe $\beta 16$," Die Wurzel, vol. 29, pp. 221-222, 1995.

[15] H. Alzer, "Ungleichungen für Mittelwerte," Archiv der Mathematik, vol. 47, no. 5, pp. 422-426, 1986.

[16] H. Alzer and S.-L. Qiu, "Inequalities for means in two variables," Archiv der Mathematik, vol. 80, no. 2, pp. 201-215, 2003.

[17] I. Costin and Gh. Toader, "A separation of some Seiffert-type means by power means," Revue d'Analyse Numérique et de Théorie de l'Approximation, vol. 41, no. 2, pp. 125-129, 2012.

[18] P. A. Hastö, "Optimal inequalities between Seiffert's mean and power means," Mathematical Inequalities \& Applications, vol. 7, no. 1, pp. 47-53, 2004.

[19] I. Costin and Gh. Toader, "Optimal evaluations of some Seifferttype means by power means," Applied Mathematics and Computation, vol. 219, no. 9, pp. 4745-4754, 2013.

[20] Y.-M. Li, M.-K. Wang, and Y.-M. Chu, "Sharp power mean bounds for Seiffert mean," Applied Mathematics, vol. 29, no. 1, pp. 101-107, 2014.

[21] A. Čizmesija, "A new sharp double inequality for generalized Heronian, harmonic and power means," Computers \& Mathematics with Applications, vol. 64, no. 4, pp. 664-671, 2012.

[22] M.-K. Wang, Y.-M. Chu, Y.-F. Qiu, and S.-L. Qiu, "An optimal power mean inequality for the complete elliptic integrals," Applied Mathematics Letters, vol. 24, no. 6, pp. 887-890, 2011.

[23] Y.-M. Chu, S.-L. Qiu, and M.-K. Wang, "Sharp inequalities involving the power mean and complete elliptic integral of the first kind," The Rocky Mountain Journal of Mathematics, vol. 43, no. 5, pp. 1489-1496, 2013.

[24] E. Neuman, "A one-parameter family of bivariate means," Journal of Mathematical Inequalities, vol. 7, no. 3, pp. 399-412, 2013.

[25] Y.-M. Chu, M.-K. Wang, and Z.-K. Wang, "A best-possible double inequality between Seiffert and harmonic means," Journal of Inequalities and Applications, vol. 2011, article 94, 7 pages, 2011.

[26] Y.-M. Chu, M.-K. Wang, and Z.-K. Wang, "Best possible inequalities among harmonic, geometric, logarithmic and Seiffert means," Mathematical Inequalities \& Applications, vol. 15, no. 2, pp. 415-422, 2012.

[27] Y.-M. Chu and S.-W. Hou, "Sharp bounds for Seiffert mean in terms of contraharmonic mean," Abstract and Applied Analysis, vol. 2012, Article ID 425175, 6 pages, 2012.

[28] M.-K. Wang, Z.-K. Wang, and Y.-M. Chu, "An optimal double inequality between geometric and identric means," Applied Mathematics Letters, vol. 25, no. 3, pp. 471-475, 2012. 


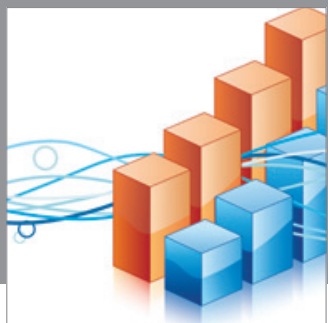

Advances in

Operations Research

mansans

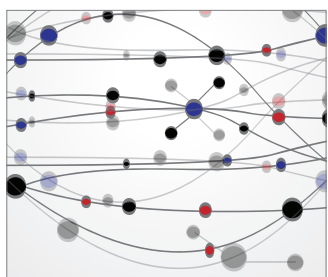

The Scientific World Journal
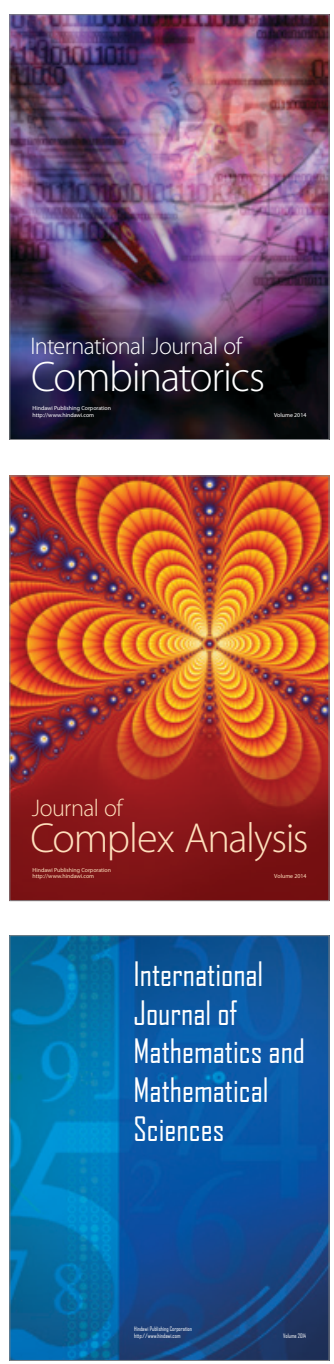
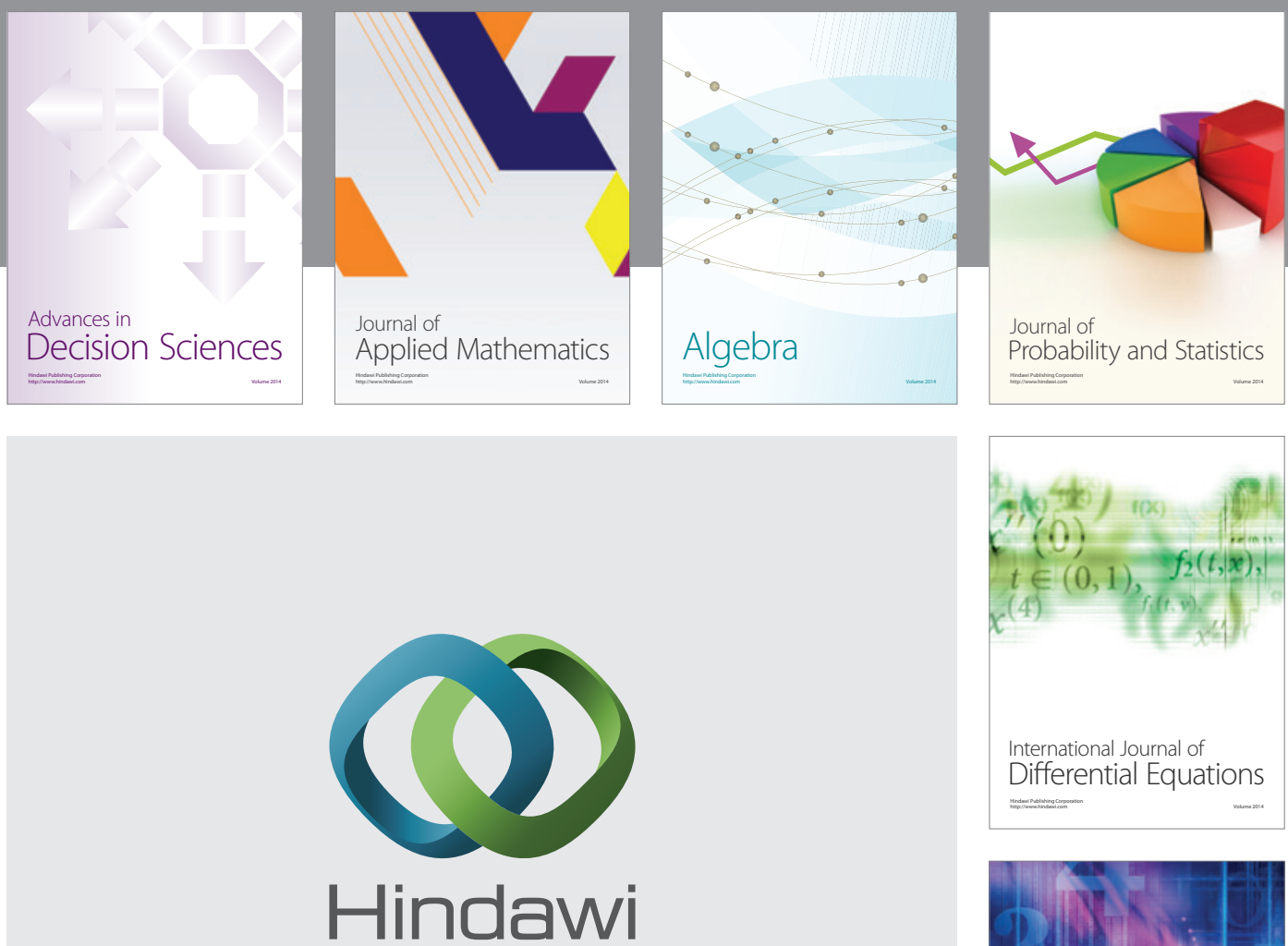

Submit your manuscripts at http://www.hindawi.com
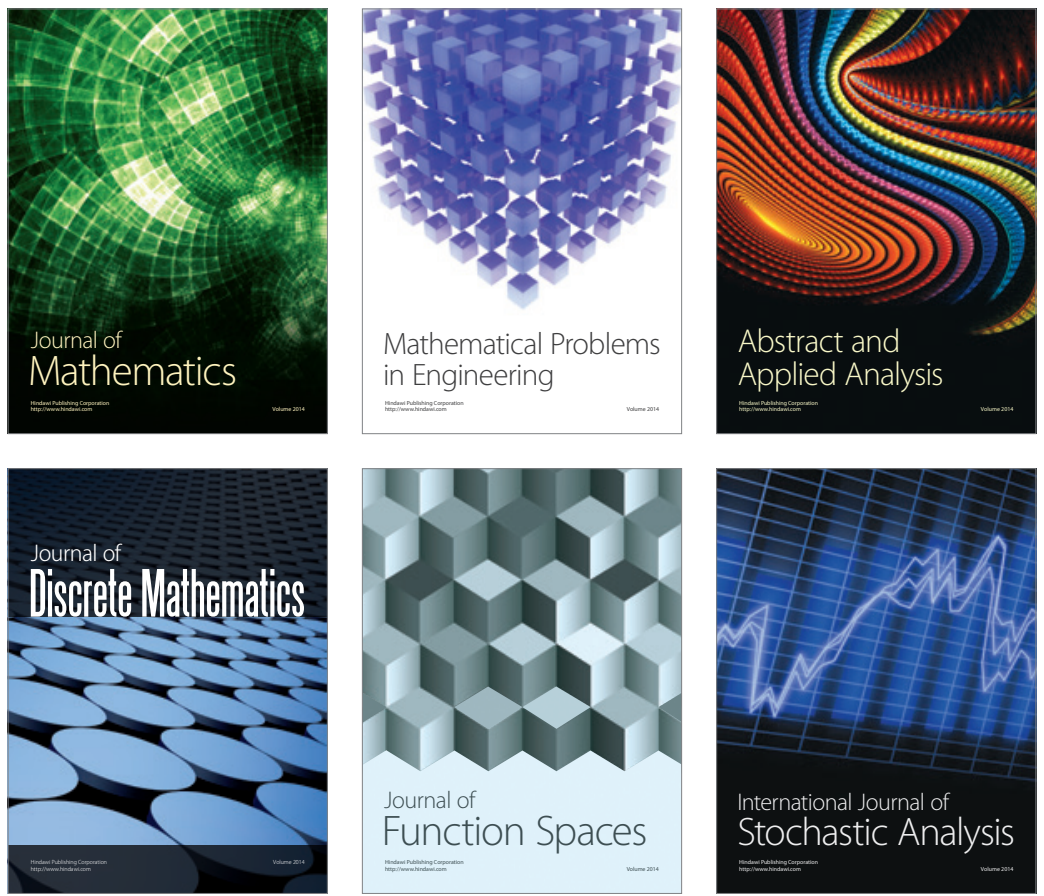

Journal of

Function Spaces

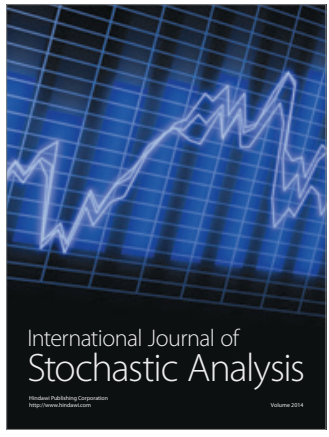

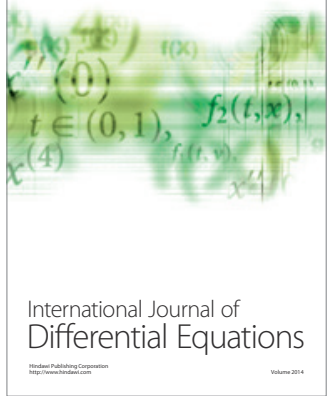
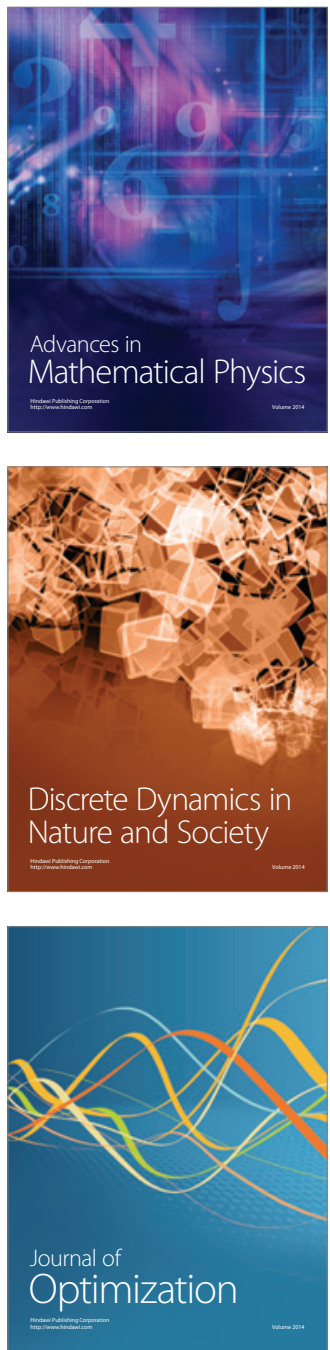